ACTA SCIENTIFIC NUTRITIONAL HEALTH (ISSN: 2582-1423)

Volume 3 Issue 8 August 2019

Short Communication

\title{
Foods that Help Keep the Doctor Away
}

\author{
Nwechi Vivian* \\ Public Health Officer at NGO, Nigeria \\ *Corresponding Author: Nwechi Vivian, Public Health Officer at NGO, Nigeria.
}

Received: July 19, 2019; Published: July 31, 2019

DOI: 10.31080/ASNH.2019.03.0393

You have probably heard that "an apple a day keeps the doctor away" but here are some foods that can also help keep the doctor away.

\section{Tomatoes}

Tomatoe is a nutrient dense super food that can be eaten raw or included in the preparation of a meal.

- Combat cancer: Tomatoes contain vitamin C which is an antioxidant which helps combat the formation of free radicals and prevent prostate cancer. Presence of lycopene may also prevent the growth of prostrate, breast, and renal cell cancer.

- Maintain blood pressure and heart: Tomatoes are rich in potassium which when combined with low sodium intake helps maintain a healthful blood pressure. Tomatoes also contain folate, this helps balance homocysteine levels. Homocysteine is an amino acid that results from protein breakdown and it also increase the risk of heart attacks and strokes.

- Constipation: Tomatoes are often described as a laxative. Tomatoes have a high water content and fiber which will help in hydration and support normal bowel movement.

- Improve eye sight: Due to the presence of vitamin A in tomatoes, vision can be improved night blindness and mascular degeneration can be prevented.

- Improves skin: Topical application of tomatoes on the skin has been known to cure severe sunburn. Tomatoes also maintain a healthy teeth, bone and hair. According to research, tomato has been found to be contained in an antiperspirant composition used for the treatment of hyperhidrosis (excessive sweating).

- Prevent gall stones: Tomato is a good source of vitamin $\mathrm{C}$ and may help cause relief from gallstones.
- $\quad$ Repair damage from smoking: Tomato does not help to quit smoking but it contains coumaric acid and chlorogenic acid that work to prevent the body from carcinogens that are produced from cigarette smoking.

Sweet potatoes

Sweet potatoes are tubers that are rich in nutrients such as vitamin C, iron, potassium, vitamin B6, calcium, phosphorous, as well as a whole bunch of other nutrients.

- Prevent inflammation: Vitamin $\mathrm{C}, \mathrm{K}$, and B6 help relieve inflammation in the intestines and digestive system. They can also be used to reduce the impact of inflammation from arthritis and gout.

- Weight management: The fiber content in potatoes act as a "bulk agent" in the digestive system, thus increasing satisfaction and reducing appetite so that a person feels full for long. Also, fiber aids to prevent constipation.

- Metabolism: Potatoes are a great source of Vitamin B6 which plays a role in energy metabolism by breaking down carbohydrates and proteins into glucose and amino acids respectively. Presence of vitamin B6 also helps to build body cells, aids in nervous system activity and athletic performance.

- Healthy bones: The presence of iron, phosphorous, calcium, magnesium and zinc in potatoes all help to build and maintain the bone structure and strength. Iron and zinc are important for production and maturation of collagen. Also, regular consumption of potatoes ensures good supply water and ions to the body.

- Prevent scurvy: Vitamin C in potatoes, help prevent scurvy.

- Sound brain: Potatoes are high in carbohydrates, therefore they promote a good level of glucose in the blood, this prevents the brain from getting fatigued, keeps cognitive activity and performance high. Also, the iron content in potatoes helps carry oxygen to the brain. 
Berries

All berries are a great source of nutrients with each berry having its own individual nutrients.

- Protect heart: Berries are good for the heart because of the presence of folate (vitamin B). It helps keep the arteries healthy by controlling blood pressure and keeping the blood from clotting. Berries are also a good source of potassium, a mineral that helps lower blood pressure. Also presence of Folate help in depression and good for pregnant women as it helps prevent fetal growth disorders and may prevent neurological birth defects.

- $\quad$ Reduce risk of parkinson's disease: Regular intake of berries reduces the risk of Parkinson's disease due to the abundance of flavonoids.

- $\quad$ Rich source of antioxidant: Blue berries are a rich source of antioxidant called Anthocyanins that may help in sharp memory even in old age. It also reduces skin wrinkling. Antioxidants protect the body from free radicals, which are unstable molecules that can damage your cells and contribute to causing cancer, thus protecting against cancer. Also, Antioxidants is linked to reduced levels of oxidized LDL (Low Density Lipoprotein).

- Weight loss: Because berries are rich in fiber, they increase feelings of fullness therefore reducing appetite.

- Fight urinary tract infection: Cranberries and blueberries help fight urinary tract infection. These berries are called anti-adhesives and help prevent growth of E. coli from binding to the wall of the bladder.

- Maintain healthy bones: Blueberries contain iron, phosphorous, calcium, magnesium, manganese, zinc, and vitamin K. Adequate intake of these minerals and vitamins contributes to building and maintaining bone structure and strength.

Enjoy a cup of berries each day as a snack, as a compliment in a meal (cereals, oat) or as a smoothie or juice.

Eggs

Egg is one of the most nutritious foods as a whole egg is packed with so many nutrients. It is true that eggs are high in cholesterol, a single egg may contain up to $212 \mathrm{mg}$ of cholesterol which is around half of the recommended dialy intake of $300 \mathrm{mg}$. However, when a dietary cholesterol is consumed, the liver reduces the amount of cholesterol it produces, to balance cholesterol level in the body. Nevertheless, excess cholesterol should be avoided especially in people with high blood level and people with genetic disorder like familiah hypercholesterolemia.

- Good source of choline: Choline is a nutrient that is unknown to most people and it is grouped under the B vitamins. It helps to build cell membranes and plays important roles in brain and memory development of the fetus, neurotransmitter synthesis and various other functions. Inadequate cholate in the body can lead to fatty liver or muscle damage. Egg yolk is the most concentrated source of choline and it may contain up to 125.5 milligrams of choline.

- Lowers risk of breast cancer: Researchers from the University of North Carolina, found the presence of choline can reduce risk of breast cancer by $24 \%$.

- Increase HDL level: Daily intake of egg can increase the level of HDL (High Density Lipoprotein) 'the good cholesterol'. This helps lowers risk of heart disease, stroke and other health problems.

- $\quad$ AID in weight loss and maintenance: The high quality of protein in eggs helps in long time satisfaction and gives energy, this prevents snacking and binge eating. Thus, reducing high calorie intake.

- Protect bones: Eggs are a good source of vitamin D which protects bones, preventing osteoporosis and rickets. Also, it contain phosphorous which provides healthy bones and teeth.

- Healthy immune system: Eggs are packed with so many nutrients such as vitamin B12, iron, zinc, phosphorous and selenium. These help to keep immune system healthy. Women also need iron due to menstruation to replenish lost blood, zinc aids the body to turn food into energy.

- Good eye sight: Lutein and Zeaxanthin in egg help to prevent macular degeneration which is the leading cause of age related blindness.

Fish

Fish includes trout, salmon, sardines, pilchards, kippers, eels, whitebait, mackerel, herring, tuna and so on. All these are rich in omega-3 polysaturated fatty acids whether canned, fresh or frozen.

- Improves child development: Consuming fish especially during the last months of pregnancy, can positively affect a child's sensory, cognitive and motor development. Although, it has not yet been determined if the same effect can be achieved during breast feeding. Also, omega-3 fat docosahexaenoic acid (DHA) is important for brain and 
eye development. It may help lower risk of asthma in children. Although fish with high mercury should be avoided, example shark, swordfish, king mackerel, tile fish etc.

- Healthy brain: Studies have shown that people who eat more fish have slower rates of medical decline and also low rate of developing Alzheimer's disease. Researchers reveal that people who eat fish every week have more gray matter (the brain's major functional tissue) in parts of the brain that regulate emotion and memory, thus helping to combat depression.

- Protects vision: Adequate consumption of fish lowers risk of AMD (Age-related Macular Degeneration), which is a leading cause of vision impairment or blindness.

- $\quad$ Good source of vitamin D: Fishes like Salmon and Herring contain high amounts of Vitamin D. Presence of vitamin D may help improve sleep and daily functioning. If you are not exposing your body to enough morning sunlight, fish might be the best alternative.

- $\quad$ Reduces risk of auto-immune disease: Auto-immune diseases are diseases that occur when the body mistakenly attacks healthy cells and tissues. Such diseases include diabetes, arthritis and multiple sclerosis. Adequate intake of omega- 3 fatty acids reduces the risk of developing these diseases.

- Always remember that eating these foods solely does not guarantee a very healthy life. They should be complimented with other nutritious foods.

Volume 3 Issue 8 August 2019

(C) All rights are reserved by Nwechi Vivian. 